This document is the accepted manuscript version of the following article:

Reichert, P., Niederberger, K., Rey, P., Helg, U., \& Haertel-Borer, S. (2019). The need for unconventional value aggregation techniques: experiences from eliciting stakeholder preferences in environmental management. EURO Journal on Decision Processes. https://doi .org/10.1007/s40070-019-00101-9

\title{
The Need for Unconventional Value Aggregation Techniques - Experiences from Eliciting Stakeholder Preferences in Environmental Management
}

Peter Reichert ${ }^{1}$, Klemens Niederberger ${ }^{2}$, Peter Rey ${ }^{3}$, Urs Helg ${ }^{4}$ and Susanne Haertel-Borer ${ }^{4}$

1 Eawag, Swiss Federal Institute for Aquatic Science and Technology, Überlandstrasse 133, 8600 Dübendorf, Switzerland

2 AQUAPLUS AG, Gotthardstrasse 30, 6300 Zug, Switzerland

3 HYDRA Institut für angewandte Hydrobiologie, Fürstenbergstr. 25, 78467 Konstanz, Germany

4 Swiss Federal Office for the Environment, FOEN, 3003 Bern, Switzerland

\section{Keywords:}

Environmental decision support; stakeholder involvement; elicitation; value function; objectives hierarchy; non-additive aggregation

Corresponding author:

Peter Reichert

Eawag, Swiss Federal Institute for Aquatic Science and Technology

Überlandstrasse 133, 8600 Dübendorf, Switzerland

peter.reichert@eawag.ch

https://orcid.org/0000-0001-7832-4257

http://www.eawag.ch/ reichert 


\section{Abstract:}

Despite the large literature about non-additive value aggregation techniques, in the large majority of applied decision support processes, additive value aggregation functions are used. The main reasons for this may be the simplicity of the approach, minimum elicitation requirements, software availability, and the appeal of the underlying preference independence concepts that may be strengthened by an adequate choice of sub-objectives and attributes. However, in an applied decision-support process, the decision maker(s) or the stakeholders decide on the subobjectives and attributes to characterize the state of a system and they have to provide information that allows the decision analyst to express their preferences as a value function of these attributes. It is the task of the decision analyst to find the parameterization and parameter values of a value function that fits best the expressed preferences. We describe a value function elicitation process for the ideal morphological state of a lake shore, performed with stakeholders from federal and cantonal authorities and from environmental consulting companies in Switzerland. This process led to the elicitation of strongly non-additive and partly even nonconcave value aggregation functions. The objective of this paper is to raise the awareness about the importance of carefully testing the assumptions underlying parameterized (often additive) value aggregation techniques during the preferences elicitation process and to be flexible regarding evaluating value functions that deviate from the often used additive aggregation scheme. This can lead to a higher confidence that additive aggregation is suitable for the specific decision problem or to the selection of alternative aggregation techniques that better represent the decision maker's preferences in case additivity is violated. 


\section{Introduction}

Decision support requires the specification of preferences for potential outcomes of a decision, the generation of decision alternatives that may have the potential to improve the fulfillment of the preferences, and the prediction of the outcomes of these alternatives. The alternatives can then be evaluated regarding their degree of fulfillment of the preferences or objectives. Such a structured approach to decision support is in the sense of value-focused thinking (Keeney, 1992) that emphasizes the discussion on what to achieve rather than on which measures to take. This stimulates a decision-making process that is as open as possible regarding the creation of alternatives that may lead to the best possible fulfillment of the pre-defined objectives.

All these three elements of decision-making are particularly difficult to implement in environmental decision support. In environmental decision support, (i) preferences should represent societal goals that, due to the diversity of perspectives, opinions and interests of individuals, do not exist in a strict sense; (ii) a limited set of alternatives is often already defined and it may not be easy to expand this set, and (iii) predictions of the behavior of environmental systems must rely on incomplete scientific knowledge and data and are thus often very uncertain.

Methods of decision analysis (see e.g. Keeney and Raiffa, 1976; von Winterfeldt and Edwards, 1986; Eisenführ et al. 2010; Clemen and Reilly, 2013) formalize "rational" decision-making. Although individuals have been shown to not necessarily take "rational" decisions in this sense (Tversky and Kahnemann, 1974; Kahneman and Tversky, 1979; Kahneman, 2003), methods of "rational" decisionmaking can be very useful to support the transparency of the decision-making process, negotiations with stakeholders, and (rational) justifications of decisions in public policy (Reichert et al. 2007, Reichert et al. 2015). These concepts can be applied qualitatively with a moderate effort to structure decision-making processes. Using more resources, they can be applied quantitatively by (i) eliciting preferences in the form of measurable value functions (Dyer and Sarin, 1979) that, if normalized to the interval $[0,1]$, describe the degree of fulfillment of objectives as functions of measurable system attributes, (ii) predicting the outcomes of decision alternatives with respect to these attributes, and (iii) evaluating the value functions at these predictions to rank the alternatives (see e.g. Keeney and Raiffa, 1976; Belton and Stewart, 2002; Eisenführ et al. 2010; Clemen and Reilly, 2013). By eliciting risk attitudes, such value functions can be transformed to utility functions (Dyer and Sarin, 1982). Using probabilistic predictions of the attributes for all decision alternatives, the alternatives can then be ranked according to expected utility to consider uncertain outcomes of decision alternatives and risk attitudes of decision makers or stakeholders. 
There are two approaches to the elicitation of value functions: One approach consists of eliciting the value function directly as a function of all relevant attributes. This is ideally done using trade-off statements (see e.g. Eisenführ et al. 2010; Reichert et al 2015; Haag et al. 2019a), discrete choice selections (Hoyos, 2010), or, in case of additive value functions, using the UTA method (JacquetLagreze and Siskos, 1982). An alternative approach consist of three steps: (i) iteratively breaking down the overall objective of the decision maker(s) into a hierarchy of mutually complementary and jointly comprehensive sub-objectives, (ii) eliciting value functions for the lowest-level sub-objectives as functions of adequate subsets of the attributes, and (iii) eliciting aggregation functions that describe the degree of fulfillment (value) of higher-level objectives as functions of the values of the directly underlying objectives. The value function of the overarching objective is then defined as the composition of the aggregation functions according to the objectives hierarchy and finally the lowest-level value functions to get a value function as a function of all attributes. As an additional benefit, this approach gets also the value functions of all sub-objectives and thus allows the decision maker to analyze which branch of the objectives hierarchy deteriorates the overarching objective (in the case that the overarching objective is poorly fulfilled). As this procedure breaks down the elicitation process into simpler sub-processes, the second approach is preferable if the value function of the overall objective is high-dimensional (depends on many attributes). The same techniques as used in the first approach can still be applied for the elicitation of the value functions of the subobjectives, except that for higher-level sub-objectives the values to aggregate must be communicated by associated attribute levels (Reichert et al. 2015).

When following the second approach, the two main challenges are to set-up a well-structured objectives hierarchy because it determines the structure of the value function, and to find adequate aggregation functions at higher levels of the hierarchy. The elicitation of the value functions at the lowest hierarchical level is comparably much easier as they usually depend only on a small number of attributes, in many cases just on a single attribute. Standard textbook approaches can be applied to elicit these value functions (e.g. Eisenführ et al. 2010).

The importance of clearly formulating objectives in decision making has been realized very early in the development of decision analysis. Keeney (1988) describes a simple procedure that starts with asking decision makers or stakeholders to list objectives they feel are important to the problem and then to separate fundamental objectives from means objectives. Questioning why something is important can strongly support this process. Hierarchy building consists of thinking of important aspects of an objective and of the comprehensiveness of the aspects already identified (Keeney, 1992; Eisenführ et al. 2010; Clemen and Reilly, 2013). Marttunen et al. (2019) provide a 
comprehensive overview of methods to structure and simplify objectives hierarchies once they have been generated.

As mentioned above, lowest-level value functions are usually relatively easy to elicit. At higher hierarchical levels, parameterizations of the aggregation functions have to be chosen that make it possible to choose parameter values so that the resulting value function well represents the preferences of the decision makers or stakeholders. In most practical applications, a weighted arithmetic mean, the so-called additive aggregation function, has been chosen to calculate the value of a higher-level objective from those of the corresponding lower-level sub-objectives. The main reasons for this may be the simplicity of the approach, minimum elicitation requirements, software availability, and the appeal of the underlying preference independence concept (see Dyer and Sarin, 1979 , for more details of the assumptions underlying measurable, additive value functions). Nevertheless, there exists a large literature about non-additive aggregation schemes or value functions e.g. multiplicative or multilinear value functions (Dyer and Sarin, 1979), aggregation functions based on the Choquet integral (Grabisch et al. 2008, Grabisch and Labreuche, 2010), or for concave value functions (Korhonen et al., 1984; Ramesh et al., 1988; Prasad et al., 1997; Korhonen et al., 2016; Argyris et al., 2014; Korhonen et al., 2017). An overview of aggregation functions can be found in Beliakov et al. (2007) or Grabisch et al. (2009). Due to the uncertainty in the elicitation process, in practice, it may be difficult to select among different parameterizations that lead to similar functional shapes. Considering the uncertainty in the elicitation process by fitting value functions of different shapes to the same elicitation data (Haag et al., 2019a) allows the decision analyst to assess the structural and parametric uncertainty of the quantified preferences and is thus a cautious way of dealing with elicitation results. In addition, the quantification of the uncertainty in the value or utility function makes it possible to consider preference uncertainty in addition to outcome uncertainty of the decision alternatives in the decision support process (Cyert and de Groot, 1979; Boutilier, 2003; Haag et al., 2019b).

Quantitatively eliciting multi-attribute value functions is a demanding and time-consuming activity. For this reason, quantitative decision support as outlined above may not be adequate for each decision. However, if there are many similar decisions with partly the same objectives hierarchies and values, it may even increase the efficiency of decision support if a value function was elicited quantitatively. The existence of such generic preferences over a significant part of an objectives hierarchy is typical in branches of environmental management, such as in surface water management. In particular, the preferences about the ecological state to be reached for surface water bodies were already traditionally specified generically (by type), although, in most present ecological assessment protocols, not in the form of value functions. To standardize methodologies 
and to facilitate the use of surface water assessment protocols in decision support processes, it makes sense to formulate these assessment protocols in the form of value functions (Reichert et al. 2007; Langhans et al. 2013; Reichert et al. 2015). This has been suggested (Reichert et al. 2011; Langhans et al. 2011), and partially achieved (Schlosser et al. 2013; Niederberger et al. 2016; Känel et al. 2018), for the Swiss surface water assessment program (Bundi et al. 2000; http://www.modulstufen-konzept.ch).

In the course of the development of the morphological lake shore assessment protocol in this framework, we identified the need for "unconventional" value aggregation techniques that may be of broader interest. It is the goal of this paper to discuss the elicitation process and the resulting value aggregation techniques to stimulate further discussion and, if adequate, application of nonadditive value or aggregation functions.

\section{Methods}

\subsection{Methodological Framework}

To support surface water management in Switzerland, as part of an overarching value function for surface water management (Schlosser et al., 2013; Reichert et al. 2015), the project had the goal of eliciting a measurable value function for the morphological assessment of lake shores. For this purpose the value scale of the lake shore assessment was linked to ecological quality classes by defining the 5 quality classes as equally spaced intervals of length 0.2 of the value scale in the interval [0,1] (Langhans et al., 2013: 0-0.2: bad; 0.2-0.4: poor; 0.4-0.6: moderate; 0.6-0.8: good; 0.8-1: high). This made the stakeholders be very familiar with the value scale and made it possible to compare and confirm elicitation results obtained by trade-offs with direct rating.

Due to the relatively large number of attributes, the value function was decided to be constructed based on an objectives hierarchy. The objectives hierarchy was constructed in an iterative process starting top-down from the main objective of reaching a good morphological state of the lake shore and discussing important aspects of objectives at each hierarchical level to construct comprehensive sets of sub-objectives. Attributes were then associated with the objectives at the lowest hierarchical level. As some of the stakeholders had been involved in the development of other lake shore assessments procedures (for Lake Constance and Lake Geneva, respectively) and were thus very familiar with the topic, the comprehensiveness of the lowest level objectives with the associated attributes could be checked and the objectives hierarchy revised.

Value functions of lowest level sub-objectives were mostly elicited by direct rating. This was possible due to the linkage of the value scale with ecological quality classes, the familiarity of the stakeholders 
with the problem, and the relative simplicity of quantifying the deterioration of the lake shore by artificial objects like a bank wall, buildings, traffic infrastructure, etc.

To complete the construction of the value function of the overall objective, aggregation functions are needed at all higher hierarchical levels. As they express the value of an objective (degree of fulfillment of the objective) as a function of the values of the associated sub-objectives, an $n$ dimensional aggregation function (aggregating $n$ sub-objectives), $F^{(n)}$, needs to have the following properties (Grabisch et al., 2011a; see also Grabisch et al., 2011b; we restrict our definition to the value interval $[0,1]$ as value functions can be re-scaled to this interval):

$$
\begin{aligned}
& F^{(n)}:[0,1]^{n} \rightarrow[0,1], \\
& F^{(n)} \text { nondecreasing in all of its arguments, } \\
& F^{(n)}(\mathbf{0})=0, F^{(n)}(\mathbf{1})=1, \quad \text { where } \mathbf{0}=(0,0, \ldots, 0), \mathbf{1}=(1,1, \ldots, 1), \\
& F^{(1)}(v)=v \quad \forall v \in[0,1] .
\end{aligned}
$$

Condition (1a) expresses that $n$ values are aggregated to a single value at the next hierarchical level and that all values are constrained to the interval $[0,1]$ (representing degrees of fulfillment of the associated objectives). Condition (1b) is important as the arguments are already values, not attributes (note that aggregation functions are only used at higher hierarchical levels; value functions at the lowest hierarchical level are functions of attributes, do not have to be monotonic, and are defined within the natural range of definition of the attributes). Condition (1c) expresses the requirement that the aggregation function does not constrain the range of possible values. Finally, condition (1d) requires that the aggregation function reduces to the identity if there is nothing to aggregate $(n=1)$. Value functions are often generically defined for arbitrary values of $n$.

In our application, we constrain the aggregation function by requesting idempotency:

$$
F^{(n)}(\boldsymbol{v})=v \forall v \in[0,1], \quad \text { where } \boldsymbol{v}=(v, v, \ldots, v)
$$

As mentioned by Grabisch et al. (2011a) this is a common restriction in multi-criteria decision analysis, as it makes sense to assume that a higher-level objective is fulfilled to the same degree as its sub-objectives, if all its sub-objectives have the same degree of fulfillment. On the other hand, we do not request concavity

$$
F^{(n)}\left(\alpha \boldsymbol{v}_{1}+(1-\alpha) \boldsymbol{v}_{2}\right) \geq \alpha F^{(n)}\left(\boldsymbol{v}_{1}\right)+(1-\alpha) F^{(n)}\left(\boldsymbol{v}_{2}\right) \quad \forall \alpha \in[0,1], \boldsymbol{v}_{1}, \boldsymbol{v}_{2} \in[0,1]^{n}
$$

or quasi-concavity

$$
F^{(n)}\left(\alpha \boldsymbol{v}_{1}+(1-\alpha) \boldsymbol{v}_{2}\right) \geq \min \left(F^{(n)}\left(\boldsymbol{v}_{1}\right), F^{(n)}\left(\boldsymbol{v}_{2}\right)\right) \quad \forall \alpha \in[0,1], \boldsymbol{v}_{1}, \boldsymbol{v}_{2} \in[0,1]^{n}
$$


of the aggregation function but we will discuss whether the elicited aggregation functions are concave. It has been discussed that this property may be desired as it limits the possibility to compensate for a poorly fulfilled sub-objective by other sub-objectives (Langhans et al., 2014). If the property (3b) is fulfilled, already during the elicitation process, alternatives with outcomes in a convex cone defined by previously expressed preferences are dominated and can be excluded from the elicitation process (see e.g. Korhonen et al., 1984; Ramesh et al., 1988; Prasad et al., 1997; Korhonen et al., 2016; Argyris et al. 2014; Korhonen et al., 2017).

\subsection{Stakeholder Involvement Process}

The stakeholders were recruited from federal and cantonal authorities and environmental consulting companies; some of them had been involved in the development and/or application of other lake shore assessment protocols. This was a standard selection process for the development of ecological assessment techniques in Switzerland.

The stakeholder involvement process extended over about 2 years and was done with the following main steps:

1. Establishment of the objectives hierarchy and attributes for the lowest-level sub-objectives.

2. Elicitation of value functions for the lowest-level objectives (by direct rating for these subobjectives, see previous section).

3. Elicitation of weights for additive aggregation functions (by comparing trade-offs).

4. Analysis of poor representation of stakeholder preferences by the additive aggregation functions and trying to resolve the issues by using non-additive, concave aggregation functions.

5. Discussion of the results for various test reaches of lake shores and with the results of other lake shore assessment protocols.

6. Extending the aggregation functions to non-concave functions to try to resolve differences in trade-offs at high or low values.

7. Comparison of evaluation results with (i) direct rating at the main objective level for several new lake sections, (ii) evaluation and review or the results for long parts of the shores of lakes with different characteristics that were not used for the construction of the value function, and (iii) analysis of completely evaluated objectives hierarchies for all possible combinations of (discrete) attribute levels.

8. Final revision until the group reached consensus about the final form and quantification of the value function.

More details to these steps and their motivation is provided in the results and discussion sections. 


\subsection{Numerical Implementation}

All objectives hierarchies and associated value functions were implemented with the aid of the $R$ package "utility" (Reichert et al. 2013; http://CRAN.r-project.org/package=utility) and are available in the R package "ecoval" (see http://CRAN.r-project.org/package=ecoval).

\section{Results: Stakeholder Involvement Process and Elicited Value Function}

We describe the construction of the objectives hierarchy and the association of attributes (section 3.1), the elicitation of the value functions of the lowest-level sub-objectives (section 3.2), and the iterative process that led to the final choice of the shape and parameters of the aggregation functions (section 3.3).

\subsection{Objectives Hierarchy and Attributes}

Fig. 1 shows the objectives hierarchy developed with the stakeholders for the target state a nearnatural lake shore morphology should reach. At the highest hierarchical level, the objective of reaching a near-natural state of the lake shore is split into the three sub-objectives of reaching a near natural shore area, a near-natural shore line, and a near natural shallow water zone. These subobjectives are further detailed with a focus on human land use and vegetation in the shore area, construction at the shore line, and bed modification and constructions in the shallow water zone.

The objectives hierarchy was developed top-down by discussing important aspects of the objectives at each hierarchical level to construct their sub-objectives. To each lowest-level sub-objective, a single attribute could be identified to be sufficient to characterize the degree of fulfillment of the sub-objective. The attributes are discrete (type of land use, type of construction, etc.). The set of attributes was compared with other lake shore assessment protocols (for Lake Constance and Lake Geneva) and served as a bottom-up check for the completeness of the objectives hierarchy. Some attributes in other assessment protocols (and related sub-objectives) that related to fish habitats were omitted as they were felt to belong to another branch of the overall objectives hierarchy of a good ecological state of the lake (the branch developed in this project was intended to assess the morphology of the lake shore only).

Already when discussing the objectives hierarchy, not all sub-objectives of an overarching objective seemed to have equal importance. No human land use was the main sub-objective of a near natural (near or farther) shore area. However, negative impacts seemed less important if there was some near natural vegetation at the shore stripe or at the border between near and farther shore areas (objectives with green border in Fig. 1). Similarly, the main objective of a near natural shore area is a 
near natural near shore area ( $0 \mathrm{~m}$ to $15 \mathrm{~m}$ from the shore line), but human land use in the farther shore area (15 $\mathrm{m}$ to $50 \mathrm{~m}$ from the shore line) should be a negatively influencing factor (objective with red border in Fig. 1).

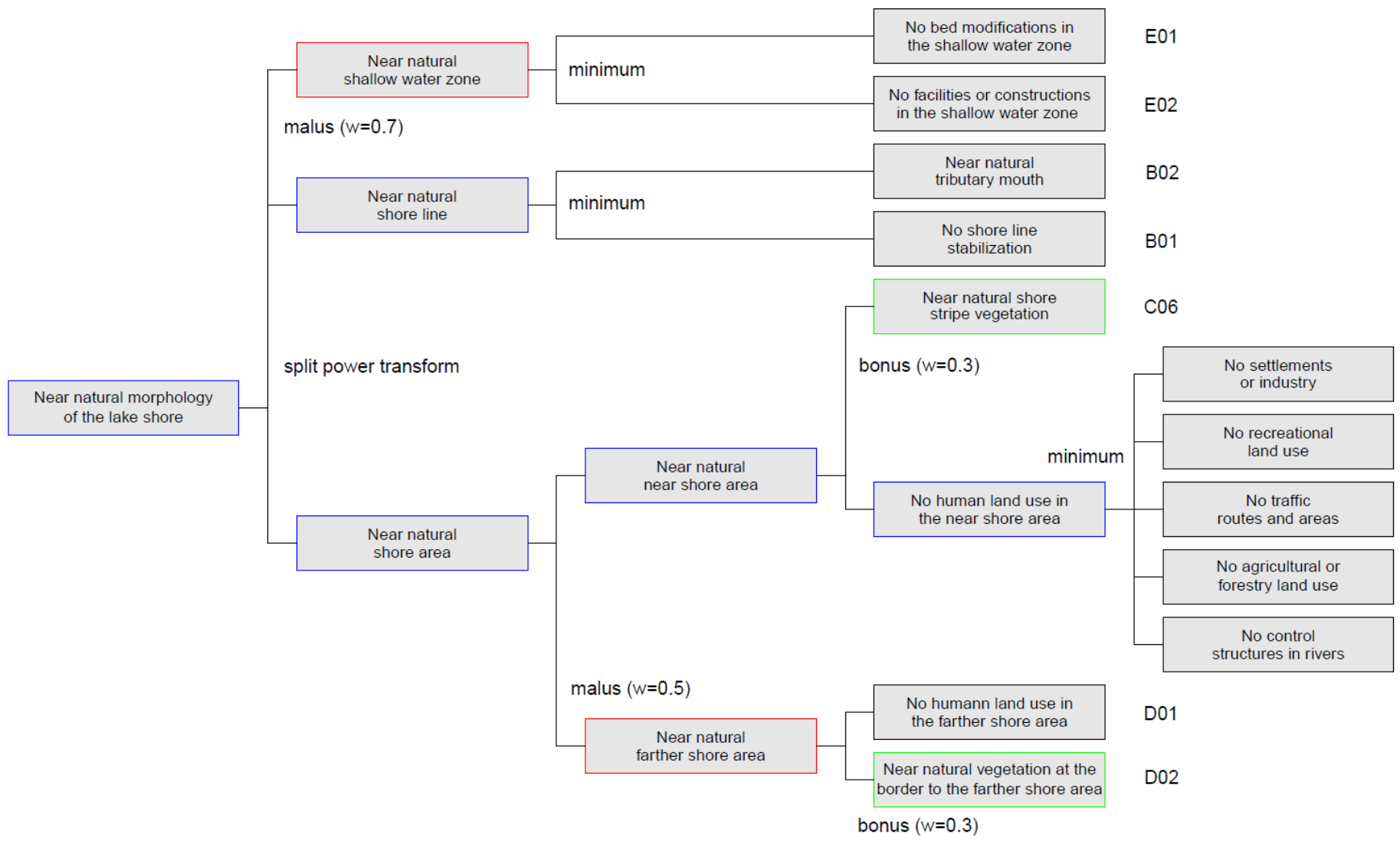

Figure 1: Objectives hierarchy for reaching a near natural morphology of the lake shore. The labels to the right of the lowest-level sub-objectives indicate the attributes, the other text outside of the boxes indicate aggregation techniques to be discussed in section 3.3.

At the top level, it was felt that the main objectives of a "near-natural shore area" and a "nearnatural shore line" both need to have a good degree of fulfillment of the objective to reach an overall good state. On the other hand, the objective of a "near-natural shallow water zone" that is typically fulfilled except in the presence of harbors or boat anchoring areas, should not generally increase the valuation by the other two objectives. However, it should decrease the overall valuation in case there is infrastructure in the shallow water zone (see objective with red border in Fig. 1).

These qualitative impressions are quantified by the aggregation functions at these nodes. This will be discussed in section 3.3 . 


\subsection{Value Functions of Lowest-Level Sub-Objectives}

Due to the familiarity of the stakeholders with the subject, the association of values with ecological states (see section 2.1 and Langhans et al., 2013), the characterization by a single attribute for each of these objectives, and the relative simplicity of quantifying morphological degradation by human land use and construction, the value functions at the lowest-level sub-objectives could be defined by direct rating. Agreement about the discrete value functions could be reached easily (see Niederberger et al., 2016 for their definition). Aggregation functions at higher levels were much more difficult to identify, as this involves the combination of diverse aspects of the higher-level objective. This required several iterations and the results are discussed in the next section.

\subsection{Aggregation Functions at Higher Hierarchy Levels}

\section{General Considerations}

The most frequently applied aggregation function is the additive aggregation defined as the weighted arithmetic mean of the values of the underlying sub-objectives:

$$
F_{\text {add }}^{(n)}(\boldsymbol{v})=\sum_{i=1}^{n} w_{i} v_{i}, \text { with } w_{i} \in[0,1], \sum_{i=1}^{n} w_{i}=1
$$

Based on past experience (e.g. Langhans et al., 2014; Haag et al., 2019a; see also Korhonen et al., 1984; Ramesh et al., 1988; Prasad et al., 1997; Korhonen et al., 2016; Argyris et al. 2014; Korhonen et al., 2017 for considerations regarding quasi-concave value functions), we also tested concave value functions, such as the minimum

$$
F_{\min }^{(n)}(\boldsymbol{v})=\min _{i} v_{i}
$$

the additive-minimum (weighted combination of additive and minimum)

$$
F_{\text {addmin }}^{(n)}(v)=\alpha \sum_{i=1}^{n} w_{i} v_{i}+(1-\alpha) \min _{i} v_{i}, \text { with } \sum_{i=1}^{n} w_{i}=1 \text { and } w_{i}, \alpha \in[0,1]
$$

and the power transformation

$$
F_{\text {pow }}^{(n)}(\boldsymbol{v})=\left(\sum_{i=1}^{n} w_{i} v_{i}^{\alpha}\right)^{1 / \alpha}, \quad \text { with } \sum_{i=1}^{n} w_{i}=1 \text { and } w_{i}, \alpha \in[0,1]
$$

aggregation functions. These functions are illustrated for $n=2$ and $w_{1}=w_{2}=\alpha=0.5$ in Fig. 2 . 

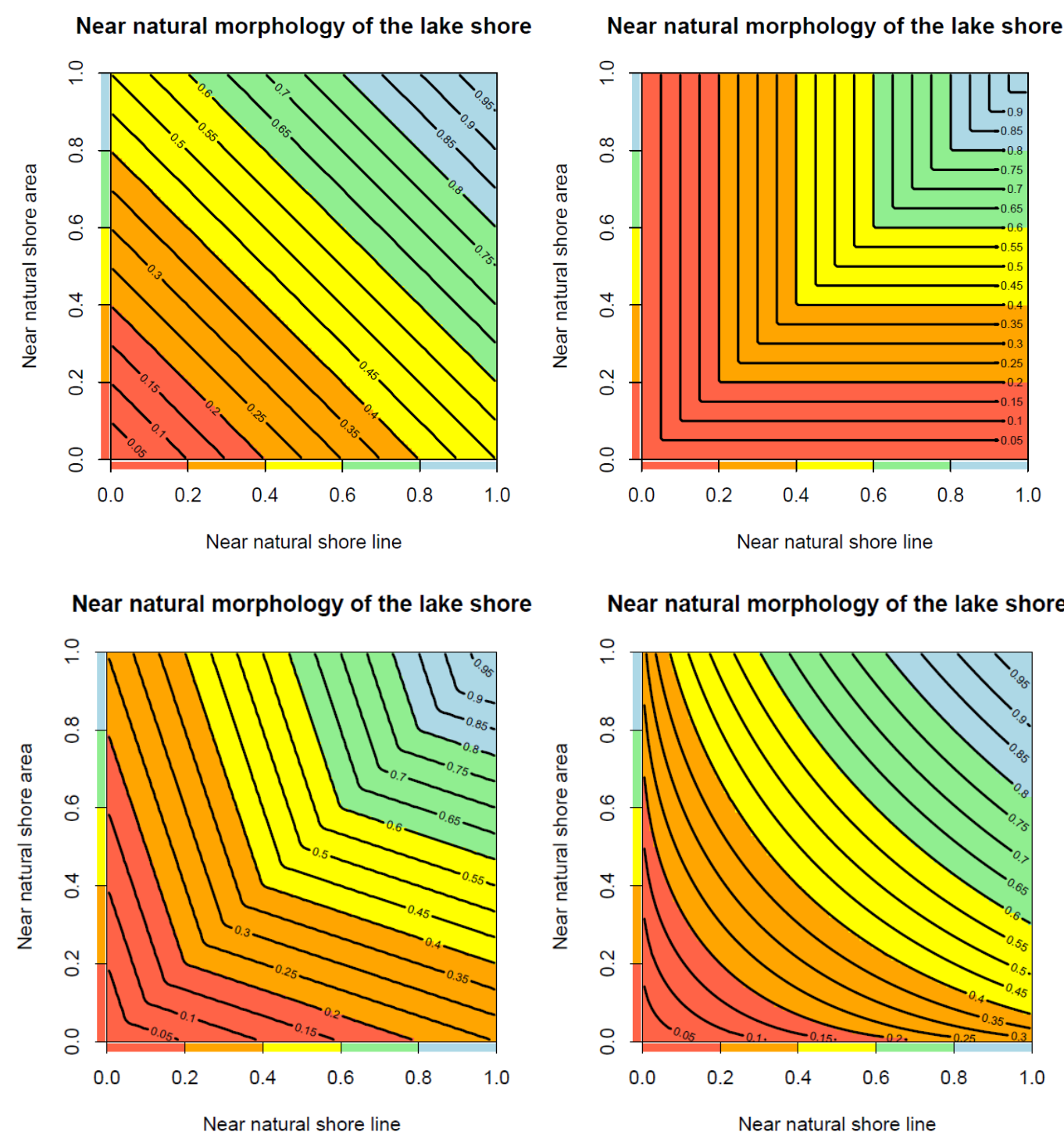

Figure 2: Illustration of additive (top left, eq. 4), minimum (top right, eq. 5), additive-minimum (bottom left, eq. 6), and power transformation (bottom right, eq. 7) aggregation functions for $n=2$ and $w_{1}=w_{2}=\alpha=0.5$. The contour lines and colors represent the aggregated value of the objective specified in the header as a function of the values of the sub-objectives labelled at the $x$ - and $y$-axes. As indicated at the axes, degrees of fulfillment are colored in red, orange, yellow, green and blue in equidistant partitions of the unit interval. The same color-coding is used for the aggregated value in the plot area.

However, at some nodes, these functions could still not sufficiently well represent the preferences of the stakeholders. The stakeholders were not content with the trade-offs when increasing one of the arguments (the value of one of the sub-objectives). These problems as well as their resolution by newly developed aggregation functions are described in the next three subsections. 


\section{Bonus Aggregation}

For some of the objectives in the objectives hierarchy, it was difficult to establish sub-objectives of similar importance. This was in particular the case for the shore area assessment that should primarily be based on the land use in the near shore area (up to $15 \mathrm{~m}$ from the shore line). However, additional elements, such as vegetation stripes and the state of the farther shore area $(15 \mathrm{~m}-50 \mathrm{~m}$ from the shore line) should be considered as well. Several attempts with additive aggregation with smaller weights for these aspects failed to represent the stakeholder's preferences. The main reason was that for most human land uses the absence of vegetation is typical and should not lead to a further deterioration of the state, whereas the presence of a (small) vegetation stripe should increase the valuation. This is not possible with additive aggregation.

To consider these preferences, a weighted mean (additive) aggregation scheme for the case that the auxiliary objective is valued higher than the main objective is combined with the consideration of only the valuation of the main objective if the auxiliary objective is valued lower than the main objective. Formally, this is expressed by

$$
F_{\text {bonus }}^{(2)}\left(v_{\mathrm{m}}, v_{\mathrm{a}}\right)=\left\{\begin{array}{cl}
v_{\mathrm{m}} & \text { for } v_{\mathrm{m}} \geq v_{\mathrm{a}} \\
w_{\mathrm{a}} v_{\mathrm{a}}+\left(1-w_{\mathrm{a}}\right) v_{\mathrm{m}} & \text { for } v_{\mathrm{m}}<v_{\mathrm{a}}
\end{array},\right.
$$

where $v_{\mathrm{m}}$ is the value of the main objective, $v_{\mathrm{a}}$ is the value of the auxiliary objective, and $w_{\mathrm{a}}$ is the weight of the auxiliary objective for the additive part of the value function. The aggregation scheme defined by equation (8) is illustrated in the left panel of Fig. 3 and numerical examples are provided in Table 1.

Table 1: $\quad$ Numerical examples of the bonus aggregation according to eq. (8) with $w_{a}=0.3$. The value of the main objective is improved if the value of the auxiliary objective is larger, otherwise the aggregated value is equal to the value of the main objective.

\begin{tabular}{c|c|c|c|l}
$v_{\mathrm{m}}$ & $v_{\mathrm{a}}$ & $w_{\mathrm{a}} v_{\mathrm{a}}+\left(1-w_{\mathrm{a}}\right) v_{\mathrm{m}}$ & $F_{\text {bonus }}^{(2)}\left(v_{\mathrm{m}}, v_{\mathrm{a}}\right)$ & comment \\
\hline $\mathbf{0 . 8}$ & $\mathbf{0 . 2}$ & 0.62 & $\mathbf{0 . 8 0}$ & $v_{\mathrm{m}} \geq v_{\mathrm{a}}:$ use $v_{\mathrm{m}}$ \\
$\mathbf{0 . 2}$ & $\mathbf{0 . 8}$ & 0.38 & $\mathbf{0 . 3 8}$ & $v_{\mathrm{m}}<v_{\mathrm{a}}:$ use weighted average
\end{tabular}

Among all tested options, this aggregation scheme best represented the preferences of the stakeholders for the sub-objectives of a "near-natural near shore area" and of a "near-natural farther shore area". The auxiliary, "bonus" sub-objectives are indicated by green borders in Fig. 1.

The weight of the additive part was chosen so that it was not possible to reach a "good state" (see Fig. 3; green value range from 0.6 to 0.8 ) if the main objective was in a "poor state" (see Fig. 3; 
orange value range from 0.2 to 0.4 ) or in a "bad state" (see Fig. 3 ; red value range from 0 to 0.2 ) even if the auxiliary objective was perfectly fulfilled (this results in $w_{a}=0.3$ ).

Note that this idea can easily be generalized for aggregations of more than two sub-objectives by aggregating all main objectives and all auxiliary objectives and then substituting these aggregated values into equation (8).

\section{Malus Aggregation}

Similarly, the state of the farther shore area should not increase the valuation (primarily of the near shore area) but decrease its value if it is lower than that of the near shore area. Formally, this is expressed by

$$
F_{\text {malus }}^{(2)}\left(v_{\mathrm{m}}, v_{\mathrm{a}}\right)=\left\{\begin{array}{cc}
v_{\mathrm{m}} & \text { for } v_{\mathrm{m}} \leq v_{\mathrm{a}} \\
w_{\mathrm{a}} v_{\mathrm{a}}+\left(1-w_{\mathrm{a}}\right) v_{\mathrm{m}} & \text { for } v_{\mathrm{m}}>v_{\mathrm{a}}
\end{array},\right.
$$

where $v_{\mathrm{m}}$ is the value of the main objective, $v_{\mathrm{a}}$ is the value of the auxiliary objective, and $w_{\mathrm{a}}$ is the weight of the auxiliary objective for the additive part of the value function. The aggregation scheme defined by equation (9) is illustrated in the right panel of Fig. 3 and numerical examples are provided in Table 2.

Table 2: $\quad$ Numerical examples of the malus aggregation according to eq. (9) with $w_{a}=0.5$. The value of the main objective is reduced if the value of the auxiliary objective is smaller, otherwise the aggregated value is equal to the value of the main objective.

\begin{tabular}{c|c|c|c|l}
$v_{\mathrm{m}}$ & $v_{\mathrm{a}}$ & $w_{\mathrm{a}} v_{\mathrm{a}}+\left(1-w_{\mathrm{a}}\right) v_{\mathrm{m}}$ & $F_{\text {malus }}^{(2)}\left(v_{\mathrm{m}}, v_{\mathrm{a}}\right)$ & comment \\
\hline $\mathbf{0 . 8}$ & $\mathbf{0 . 2}$ & 0.5 & $\mathbf{0 . 5}$ & $v_{\mathrm{m}}>v_{\mathrm{a}}:$ use weighted average \\
$\mathbf{0 . 2}$ & $\mathbf{0 . 8}$ & 0.5 & $\mathbf{0 . 2}$ & $v_{\mathrm{m}} \leq v_{\mathrm{a}}:$ use $v_{\mathrm{m}}$
\end{tabular}

Among all tested options, this aggregation scheme best represented the preferences of the stakeholders for the sub-objectives of a "near-natural shore area" and for aggregating the aggregate of the "near-natural shore area" and the "near-natural shore line" with the "near-natural shallow water zone" (note that this would be better represented by an additional node in the objectives hierarchy that aggregates the "near-natural shore area" and the "near-natural shore line", but this was not in the sense of the stakeholders; the objectives hierarchy shown in Fig. 1 is also correct, just that it needs a more complex aggregation function at the top level). The auxiliary, "malus" subobjective is indicated by a red border in Fig. 1. Note that with nearly any other aggregation method (except the minimum aggregation), it would not be possible to reach a value of zero if the shore line and the shore area are fully degraded (value of zero), but the shallow water zone is not degraded (value of one). It was a high priority for the stakeholder not to "dilute" the assessment of the shore 
area and the shore line in this sense with the shallow water zone as this is primarily a lake shore morphology assessment method and the shallow water zone is often not degraded.
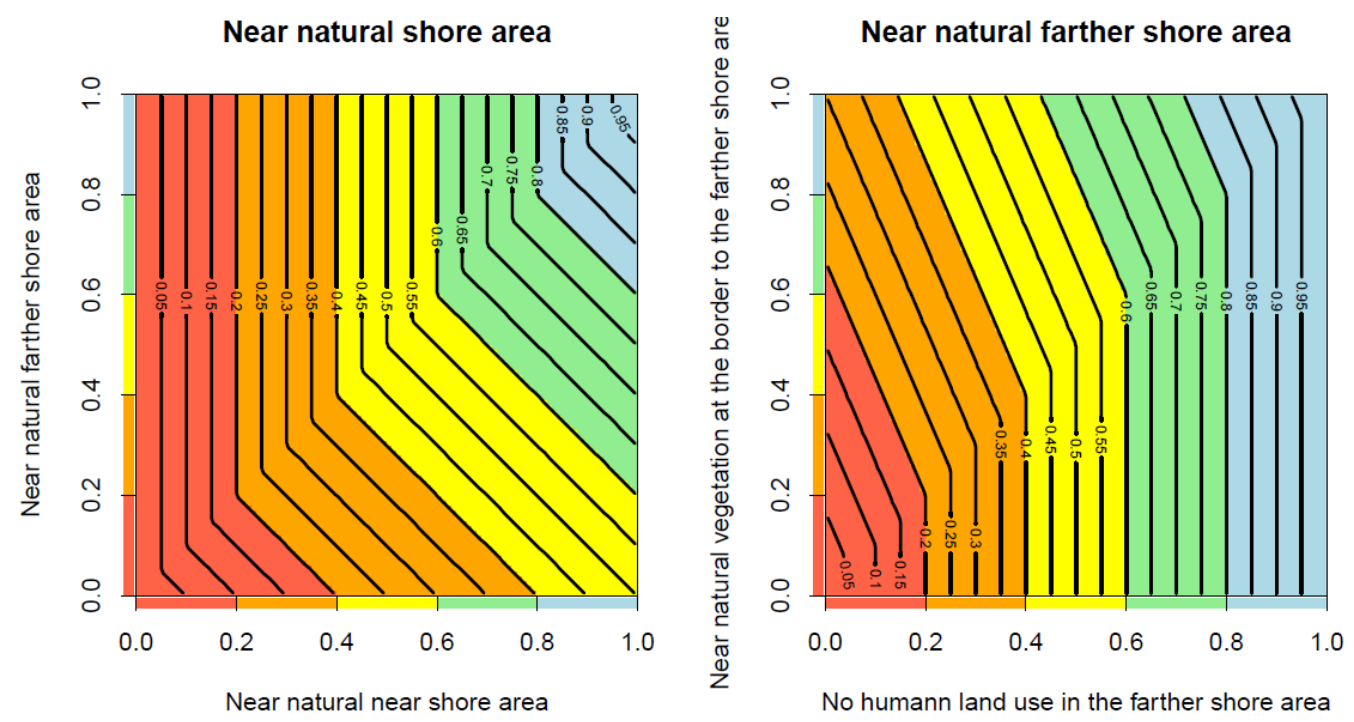

Figure 3: Illustration of a malus (left, eq. 9 for $w_{a}=0.5$ ) and a bonus (right, eq. 8 for $w_{a}=0.3$ ) aggregation function.

Note that this idea can easily be generalized for aggregations of more than two sub-objectives by aggregating all main objectives and all auxiliary objectives and then substituting these aggregated values into equation (9).

Higher weights ( $w=0.5$ and $w=0.7$, respectively for the two cases of malus aggregation, see Fig. 1 and Fig. 3) for the auxiliary objective were chosen than for the bonus aggregation as it was felt that the auxiliary objective should lead to a strong deterioration of the state, in particular in case of a modified shallow water zone of the lake.

\section{Split Power Transform Aggregation}

The aggregation function at the top level, aggregating the values of the shore area, the shore line, and the shallow water zone to the value of the overall objective of reaching a near-natural lake shore, turned out to be most difficult and required several attempts to reach consensus.

Additive aggregation did not seem adequate for two reasons: First, construction is mostly limited to the shore line and the shore area. With similar weights for the three sub-objectives, a good evaluation of the shallow water zone (which is the typical case) would make it impossible to reach a very low overall value, even if the shore area and the shore line would have values of zero (which should lead to a very low overall value). Reducing the weight of the shallow water zone does not solve the problem as then a low value of the shallow water zone does not sufficiently deteriorate the overall value. Second, stakeholders wanted the requirement that both shore line and shore area 
need to have high values in order to allow for an overall high value. These sub-objectives are complementary and both of high importance.

The problem with the shallow water zone was solved by using a "malus" aggregation with the aggregated result of the shore line and the shore area as a main objective and the shallow water zone as an auxiliary objective as discussed in the subsection on "malus" aggregation above.

This reduced the problem to aggregating the "near-natural shore area" with the "near-natural shore line". The argument that both need a high degree of fulfillment to lead to a good aggregated state was very similar to the argument in Langhans et al. (2014) and Haag et al. (2019a) that led to aggregation functions of shapes as shown in the bottom panels of Fig. 2. For this reason, we proceeded with such shapes for the next round of stakeholder discussion. These aggregation functions seemed to represent the preferences of the stakeholders very well for good valuations of the sub-objectives (being restrictive for a good overall value, see "upper right corner" of the diagrams in the bottom row of Fig. 2). However, these aggregation functions did not represent the stakeholder's preferences in the "lower left corner": if both values are low, increasing the value of one objective should lead to a more pronounced increase in the overall value.

This deficit can only be remedied by keeping or increasing the curvature of the iso-value lines in the "upper right corner" of the aggregation function shown in the bottom right panel of Fig. 2, but reversing the curvature in the "lower left corner". The aggregation function in the bottom right panel of Fig. 2 was constructed as a member of the general class of idempotent value functions obtained by transforming the values of the sub-objectives, taking the weighted mean of the transformed values, and inversely-transform the results. Formally, this important class of non-additive value functions (if the transformation, $g$, is not the identity), is given by

$$
F_{g}^{(n)}(\boldsymbol{v})=g^{-1}\left(\sum_{i=1}^{n} w_{i} g\left(v_{i}\right)\right) \quad, \quad w_{i} \in[0,1], \sum_{i=1}^{n} w_{i}=1,
$$

with a suitable monotonic transformation $g$. In particular, the aggregation function in the bottom right panel of Fig. 2 was produced with the transformation

$$
g_{\text {pow }}(v)=v^{\alpha}, g_{\text {pow }}^{-1}(v)=v^{\frac{1}{\alpha}}
$$

with $\alpha=1 / 2$ and equal weights in equation (10). This transformation is shown in Fig. 4. 

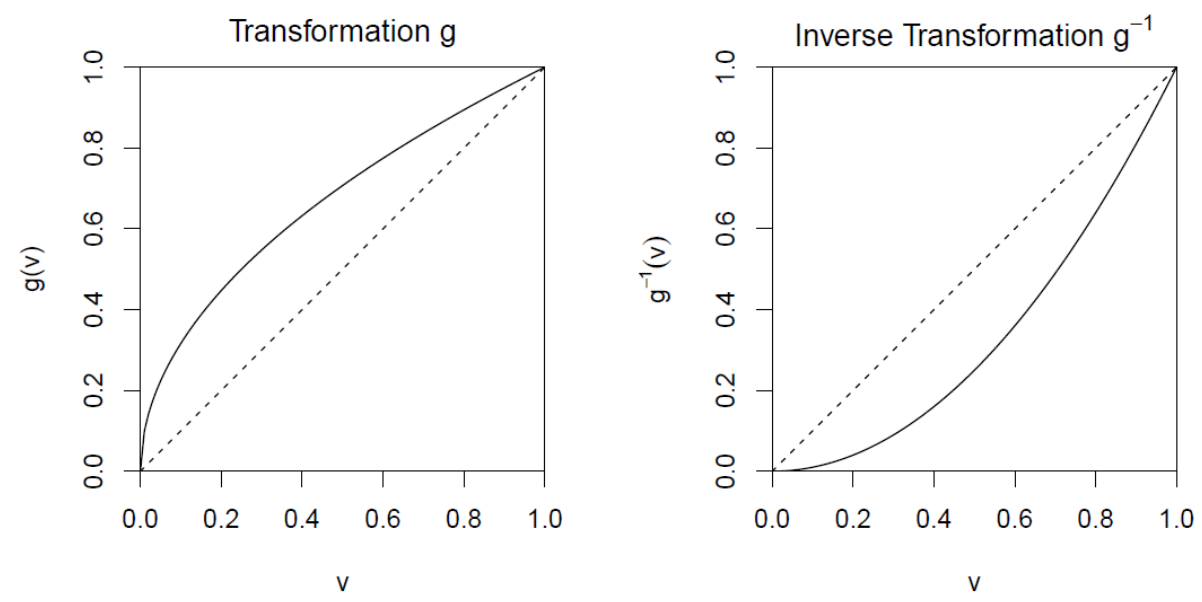

Figure 4: Transformation (11) applied to the values before aggregation (left) and inverse transformation applied to the results of the additive aggregation of the transformed values (right) to get the aggregation shown in the bottom right panel of Fig. 2.

To get the behavior that better matches the preferences of the stakeholders, we revert the curvature of the transformation as follows:

$$
g_{\text {split }}(v)=\left\{\begin{array}{cl}
\frac{1}{2}(2 v)^{\alpha} & \text { for } v \leq \frac{1}{2} \\
1-\frac{1}{2}(2(1-v))^{\alpha} & \text { for } v \geq \frac{1}{2}
\end{array} \quad, \quad g_{\text {split }}{ }^{-1}(v)=\left\{\begin{array}{cc}
\frac{1}{2}(2 v)^{\frac{1}{\alpha}} & \text { for } v \leq \frac{1}{2} \\
1-\frac{1}{2}(2(1-v))^{\frac{1}{\alpha}} & \text { for } v \geq \frac{1}{2}
\end{array}\right.\right.
$$

Fig. 5 shows this transformation for $\alpha=2$.
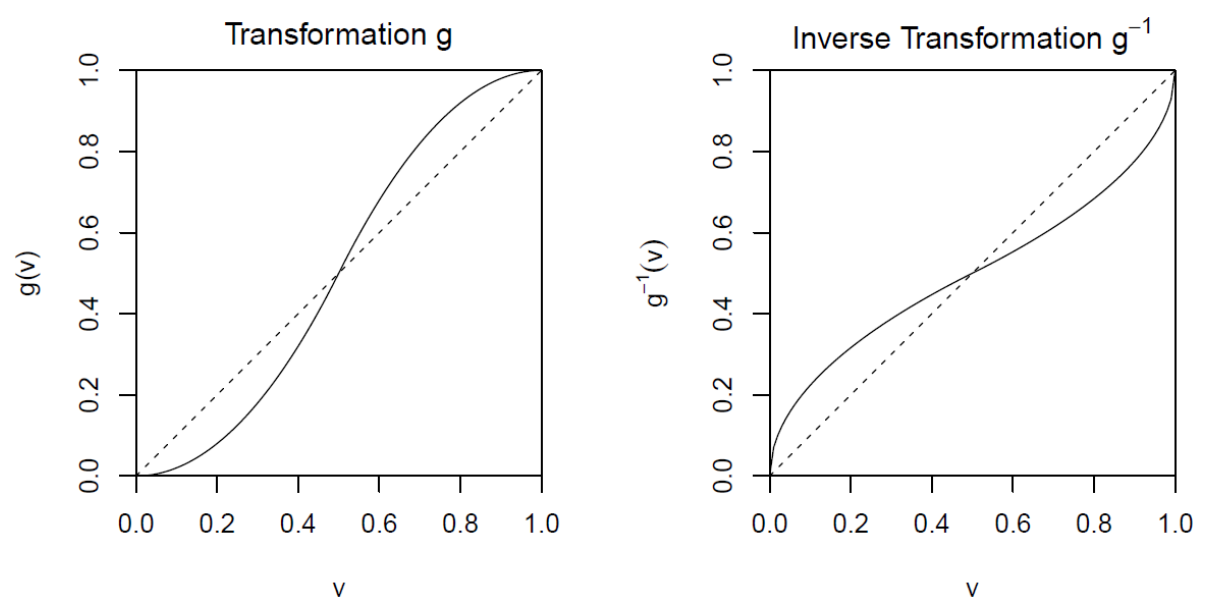

Figure 5: Transformation (12) applied to the values before aggregation (left) and inverse transformation applied to the results of the additive aggregation of the transformed values (right) to get the aggregation shown in the right panel of Fig. 6 . 

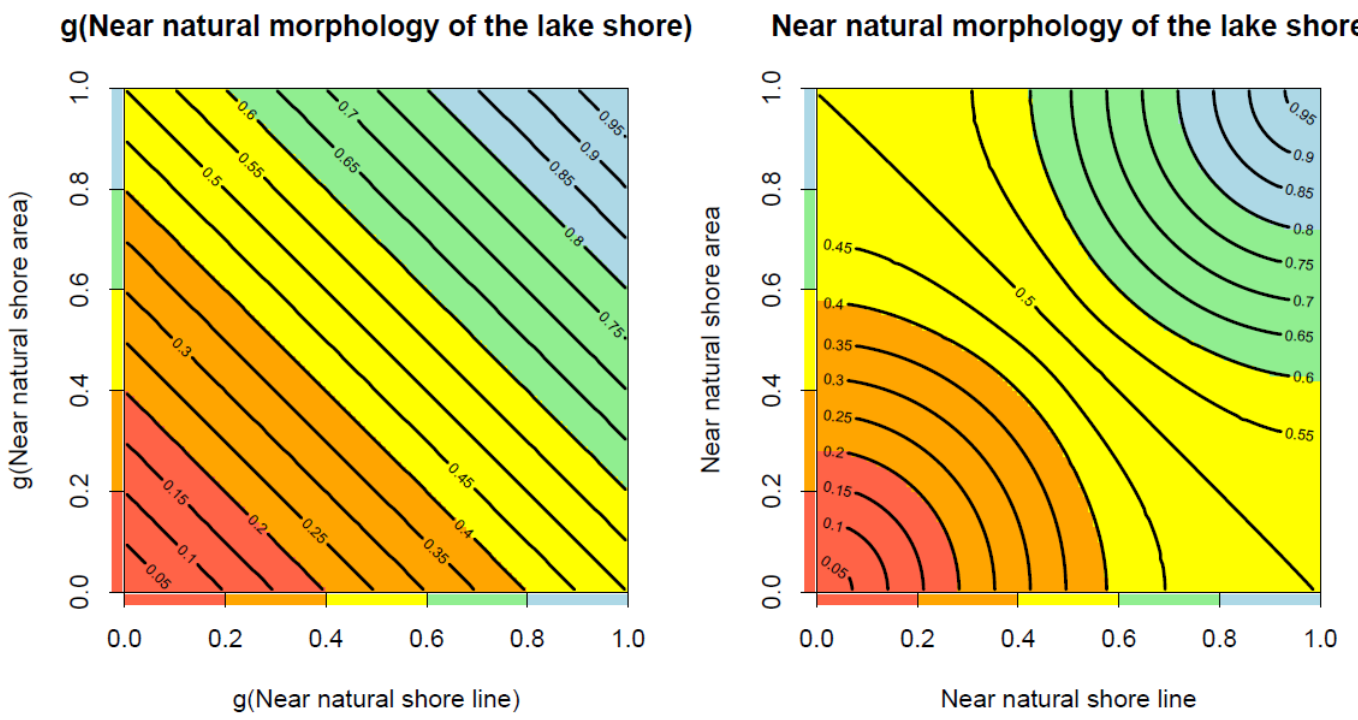

Figure 6: Illustration of additive aggregation of the split-power transformed values (left) and the resulting aggregation formulated with the non-transformed values according to the equations 10 and 12 for $n=2, w_{1}=w_{2}=0.5$. and $\alpha=2$.

This aggregation scheme is shown in the right panel of Fig. 6 and numerical examples are provided in Table 3.

Table 3: Numerical examples of the split power transformed aggregation according to eqs. (10) and (12) with $w_{1}=w_{2}=0.5$. and $\alpha=2$.

\begin{tabular}{c|c|c|c|c|c}
$v_{1}$ & $v_{2}$ & $g_{\text {split }}\left(v_{1}\right)$ & $g_{\text {split }}\left(v_{2}\right)$ & $v_{\text {aux }}=w_{1} g_{\text {split }}\left(v_{1}\right)+w_{2} g_{\text {split }}\left(v_{2}\right)$ & $F_{g_{\text {split }}}^{(2)}\left(v_{1}, v_{2}\right)=g_{\text {split }}^{-1}\left(v_{\text {aux }}\right)$ \\
\hline $\mathbf{0 . 8}$ & $\mathbf{0 . 7}$ & 0.92 & 0.82 & 0.87 & $\mathbf{0 . 7 4 5}$ \\
$\mathbf{0 . 2}$ & $\mathbf{0 . 4}$ & 0.08 & 0.32 & 0.20 & $\mathbf{0 . 3 1 6}$
\end{tabular}

The final aggregation at the top level consisted of the aggregation shown by the right panel of Fig. 6 (equations 10 and 12 with $\alpha=2$ and all weights equal) for the sub-objectives of a "near-natural shore line" and a "near-natural shore area) and a "malus" aggregation of this result with the evaluation of the shallow water zone.

\section{Minimum Aggregation}

The aggregation techniques discussed in the previous section were combined with 3 minimum aggregations (see Fig. 1) for cases where this seem adequate ("near natural shallow water zone"), where it is not relevant ("near natural shore line", as there is either a shore line or a tributary in a given section and thus only one sub-objective will be evaluated), or where the valuation of the subobjectives is already done in value units of the overarching objective ("no human land use in the near 
shore area", e.g. the value range of the sub-objective "no agricultural or forestry land use" was restricted to the interval $[0.25,1]$ rather than $[0,1]$ to reflect the fact that agricultural use is less damaging than the worst cases of industrial use).

\section{Overall Value Function}

Omitting the parameters as function arguments and using the same symbol, $v$, for all lowest-level, single attribute value functions (they are then only distinguished by their argument and documented in Niederberger et al., 2016), we end with the following value function:

$$
\begin{aligned}
& v(\boldsymbol{a})=v\left(a_{\mathrm{E} 01}, a_{\mathrm{E} 02}, a_{\mathrm{B} 02}, a_{\mathrm{B} 01}, a_{\mathrm{C} 06}, a_{\mathrm{C} 01}, a_{\mathrm{C} 02}, a_{\mathrm{C} 03}, a_{\mathrm{C} 04}, a_{\mathrm{C} 05}, a_{\mathrm{D} 01}, a_{\mathrm{D} 02}\right)= \\
& F_{\text {malus }}^{(2)}\left(F_{g_{\mathrm{split}}}^{(2)}\left(\begin{array}{c}
F_{\min }^{(2)}\left(v\left(a_{\mathrm{B} 01}\right), v\left(a_{\mathrm{B} 02}\right)\right), \\
\left.F_{\text {malus }}^{(2)}\left(\begin{array}{c}
F_{\text {bonus }}^{(2)}\left(F_{\text {min }}^{(5)}\left(v\left(a_{\mathrm{C} 01}\right), v\left(a_{\mathrm{C} 02}\right), v\left(a_{\mathrm{C} 03}\right), v\left(a_{\mathrm{C} 04}\right), v\left(a_{\mathrm{C} 05}\right)\right), v\left(a_{\mathrm{C} 06}\right)\right) \\
F_{\text {bonus }}^{(2)}\left(v\left(a_{\mathrm{D} 01}\right), v\left(a_{\mathrm{D} 02}\right)\right)
\end{array}\right)\right), \\
F_{\text {min }}^{(2)}\left(v\left(a_{\mathrm{E} 01}\right), v\left(a_{\mathrm{E} 02}\right)\right)
\end{array}\right)\right)
\end{aligned}
$$

(Note that, due to space restrictions, some argument lists are continued on subsequent lines; in any case all arguments are comma-separated lists between the corresponding bracket pair.) The function given by equation (13) expresses the degree of achievement of the overall objective, a good morphological state of the lake shore, as a function of the 12 attributes indicated on the right-hand side of the lowest-level sub-objectives in Fig. 1. The non-trivial arguments in the functions in equation (13) are the value functions of the sub-objectives in Fig. 1.

\section{Validation}

This value function with the parameters mentioned above and indicated in Fig. 1 then passed the three levels of validation listed in step 7 of the methodology (see section 2.2):

- The stakeholders were asked for direct rating of the overall morphological state of selected lake shore sections that were not used for the elicitation of the value function and the results were compared with results from applying the elicited value function (13) to these sections.

- The stakeholders reviewed the application of the value function to large sections of lake shores from lakes with different characteristics and agreed with the outcomes.

- Based on all possible combinations of the discrete attributes, a file was created with all possible objectives hierarchies, sorted with decreasing value of the overall objective and reviewed by the stakeholders. In particular, they could check whether they are (nearly) indifferent between different combinations of values of sub-objectives that led to similar values of the overall objective. 
This extensive testing with new and artificial data led finally to a high confidence of the stakeholders about the chosen approach.

\section{Discussion}

This study was part of the strategy to introduce formal decision analysis methods into surface water management in Switzerland. In particular, our vision is to formulate ecological assessment procedures for surface water bodies as branches of an overarching value function for surface water management. This would have the advantages of (i) using the same framework for all ecological assessment procedures, (ii) transparently communicating the fulfillment of the main objective and its sub-objectives in an objectives hierarchy to facilitate the identification of poorly fulfilled subobjectives that cause a deterioration of the overall objective, and (iii) building a first step to supporting water management more comprehensively with decision analysis methods. Such a process takes a lot of time and discussion ( 5 to 10 years for the concepts and much more if all ecological assessment procedures would be adapted). In our case, the stakeholders were convinced by the benefits through the transparency of the process and the clarity of representing the degree of fulfillment of objectives across all relevant hierarchical levels.

The objectives hierarchy and the corresponding value function for lake shore morphology assessment, as discussed in this paper, is only a small branch of such an overarching surface water management objectives hierarchy. It was developed in a very constructive stakeholder involvement process in about 2 years.

The definition of the value functions of the lowest-level sub-objectives by direct rating is an exceptional case and was possible for the following reasons: the familiarity of the stakeholders with the subject, the association of values with ecological states (see section 2.1 and Langhans et al., 2013), the characterization by a single attribute for each of these objectives, and the relative simplicity of quantifying morphological degradation by human land use and construction. This methodology should not be transferred to other decision situations as usually, value functions would better be elicited based on established procedures in the literature (see e.g. Eisenführ et al., 2010) avoiding direct rating. Due to the small number of attributes (often, as in our case, just one per lowest-level sub-objective) and the narrow focus of the lowest-level sub-objectives, this is typically not a very difficult preference elicitation problem. However, finding appropriate aggregation schemes at higher hierarchical levels is much more challenging. The strong preferences of the stakeholders about objectives and their aggregation led to interesting non-additive aggregation techniques as discussed below. 
The discussion about the aggregation of the assessment of the lake shore line and the lake shore area first seemed to follow the typical case of non-additivity by requiring a concave aggregation technique that "punishes" poorly fulfilled individual sub-objectives more strongly than in additive aggregation. Examples of such aggregation techniques are shown in the bottom row of Fig. 2 . However, such concave aggregation functions lead only to a weak increase of the aggregated values if both sub-objectives are in a poor state and one of them is improved. This did not match the preferences of the stakeholders. For this reason, the aggregation function shown in the right panel of Fig. 6 was introduced that combines the strong punishment of deteriorating objectives at high values with a relatively strong rewarding of improving objectives at low values. This behavior matched the preferences of the stakeholders for these objectives.

The other unconventional aggregation technique can best be discussed with the aggregation of the combined shore line and shore area assessment with the assessment of the shallow water zone. As the shallow water zone is mostly in a good condition, it should not improve a bad valuation of shore line and shore area because then a very bad state (value 0 ) would not be possible if the shallow water zone is in a good state. On the other hand, a heavily modified shallow water zone should lead to a strong deterioration of the overall assessment even if the shore line and shore area are in a good state. Mapping these conditions in a value function led to the "malus" aggregation technique that considers the auxiliary sub-objective (with additive aggregation) only if its value is smaller than the value of the main objective (see left panel of Fig. 3) and accepts the value of the main objective as the aggregated objective otherwise (see left panel of Fig. 3).

Similarly, for "bonus" aggregation, the auxiliary objective is only considered if its value is larger than the value of the main objective and accepts the value of the main objective as the aggregated objective otherwise (see right panel of Fig. 3).

\section{Conclusions}

The development of an ecological assessment procedure of the morphology of lake shores in the form of a hierarchically structured measurable value function led to the need for two types of nonadditive value aggregation functions that are not typically used in decision analysis.

One of these ("split power transform" aggregation) combines a concave aggregation function for large values of the sub-objectives with a convex aggregation function for small values of the subobjectives. The main motivation for this shape was to have strict requirements for a good overall state but still allow for substantial improvements of a bad state if the fulfillment of one of the subobjectives improves. 
The other type of non-additive aggregation ("bonus" and "malus" aggregation) was to distinguish a main and an auxiliary sub-objective and consider the auxiliary sub-objective only in case that its value is higher (bonus) or smaller (malus) than the value of the main sub-objective.

The reasons for the construction of these value functions are discussed in more detail in the paper and the value functions are formally discussed. We hope that this discussion raises the awareness of carefully testing the assumptions underlying parameterized (often additive) value aggregation techniques during the preferences elicitation process and to be flexible regarding value functions that deviate from the often used additive aggregation scheme. This can lead to a higher confidence that additive aggregation is suitable for the specific decision problem or to the selection of alternative aggregation techniques that better represent the decision maker's preferences in case additivity is violated. The elicitation process described in this paper and the resulting value functions may inspire this process of testing alternative aggregation techniques.

This could be done by extending a "standard elicitation process" by (i) carefully following the argumentation of the decision makers or stakeholders and consider also non-quantitative arguments to find appropriate parameterizations of the value or aggregation functions (the value functions developed in this project may inspire such a process), (ii) trying to match or even better to statistically fit different value and/or aggregation functions (avoiding unnecessary limitations) to the elicitation results and compare the quality of and the estimated uncertainty of the parameters, and (iii) validate the elicited value function with additional potential outcomes in a second round of elicitation interviews.

\section{Acknowledgments}

We thank the stakeholder group for the development of the ecological assessment of lake shore morphology, Heinz Ehmann, Christoph Iseli, Brigitte Lods-Crozet, Robert Lovas, Patrick Steinmann, Markus Zeh for their constructive ideas and quantitative assessments, and Evi Binderheim, Christian Michel, Jacqueline Schlosser, Nele Schuwirth and Gregor Thomas for stimulating discussions.

\section{Supporting Information}

An Excel spreadsheet and a R script for evaluating the numerical aggregation examples in Tables $1-3$ are provided as supporting information. 


\section{References}

Argyris, N., Morton, A., \& Figueira, J. R. (2014). CUT: A multicriteria approach for concavifiable preferences. Operations Research 62(3), 633-642. https://doi.org/10.1287/opre.2014.1274

Beliakov G., Pradera A., Calvo T. (2007). Aggregation functions: a guide for practitioners. Springer.

Belton V. and Stewart T.J. (2002). Multiple criteria decision analysis: an integrated approach. Boston: Kluwer Academic Publishers.

Boutilier, C. (2003). On the foundations of expected expected utility. In: Proceedings of the 18th international joint conference on Artificial Intelligence. Acapulco, Mexico: Morgan Kaufmann Publishers Inc., 285-290.

Bundi, U., Peter, A., Frutiger, A., Hütte, M., Liechti, P., Sieber, U. (2000). Scientific base and modular concept for comprehensive assessment of streams in Switzerland. Hydrobiologia 422/423, 477487. https://doi.org/10.1023/A:1017071427716

Clemen, R.T., Reilly, T. (2013). Making Hard Decisions - With Decision Tools. South-Western, third ed. Cengage Learning.

Cyert, R.M. and de Groot, M.H. (1979). Adaptive utility. In: Allais, M. and Hagen, O. eds. Expected Utility Hypotheses and the Allais Paradox. D. Reidel, Dordrecht. 223-241.

Dyer J.S. and Sarin R.K. (1979). Measurable multiattribute value functions. Oper Res 27, 810-22. https://doi.org/10.1287/opre.27.4.810

Dyer, J.S. and Sarin, R.K. (1982). Relative risk aversion. Manag. Sci. 28 (8), 875-886. https://doi.org/10.1287/mnsc.28.8.875

Eisenführ, F., Weber, M. and Langer, T. (2010). Rational Decision Making. Springer, Berlin, Germany.

Grabisch, M., Kojadinovic, I., Meyer P. (2008). A review of methods for capacity identifi- cation in Choquet integral based multi-attribute utility theory applications of the Kappalab R package. Eur J Oper Res 186, 766-85. https://doi.org/10.1016/j.ejor.2007.02.025

Grabisch, M. and Labreuche, C. (2010). A decade of application of the Choquet and Sugeno integrals in multi-criteria decision aid. Ann Oper Res 175, 247-86. https://doi.org/10.1007/s10479-009$\underline{0655-8}$

Grabisch, M., Marichal, J.-L., Mesiar, R., Pap, E. (2009). Aggregation functions. Cambridge: Cambridge University Press.

Grabisch, M., Marichal, J.-L., Mesiar, R. and Pap, E. (2011a). Aggregation functions: Means. Information Sciences 181, 1-22. https://doi.org/10.1016/i.ins.2010.08.043 
Grabisch, M., Marichal, J.-L., Mesiar, R. and Pap, E. (2011b). Aggregation functions: Construction methods, conjunctive, disjunctive and mixed classes. Information Sciences 181, 23-43.

https://doi.org/10.1016/i.ins.2010.08.040

Haag, F., Lienert, J., Schuwirth, N. and Reichert, P. (2019a). Identifying non-additive multi-attribute value functions based on uncertain indifference statements. Omega 95, 49-67. https://doi.org/10.1016/j.omega.2018.05.011

Haag, F., Reichert, P., Maurer, M., Lienert, J. (2019b) Integrating uncertainty of preferences and predictions in decision models: an application to regional wastewater planning. Journal of Environmental Management 252. https://doi.org/10.1016/i.jenvman.2019.109652.

Hoyos, D. (2010). The state of the art of environmental valuation with discrete choice experiments. Ecological Economics 69. 1595-1603. https://doi.org/10.1016/j.ecolecon.2010.04.011

Jacquet-Lagreze, E. and Siskos, J. (1982). Assessing a set of additive utility functions for multicriteria decision-making, the UTA method. European Journal of Operational Research 10, 151-164. https://doi.org/10.1016/0377-2217(82)90155-2

Kahneman, D. and Tversky, A. (1979). Prospect theory: an analysis of decision under risk. Econometrica 47(2), 263-291. https://EconPapers.repec.org/RePEc:ecm:emetrp:v:47:y:1979:i:2:p:263-91

Kahneman, D. (2003). A perspective on judgment and choice - Mapping bounded rationality. American Psychologist 58(9), 697-720. https://doi.org/10.1037/0003-066x.58.9.697

Känel, B., Michel, C. and Reichert, P. (2017). Methoden zur Untersuchung und Beurteilung der Fliessgewässer. Makrophyten - Stufe F (flächendeckend) und Stufe S (systembezogen). Entwurf. Bundesamt für Umwelt, Bern. Umwelt-Vollzug, $119 \mathrm{~S}$.

https://www.dora.lib4ri.ch/eawag/islandora/object/eawag:17319

Keeney, R.L. (1988). Building models of values. European Journal of Operational Research 37, 149157. https://doi.org/10.1016/0377-2217(88)90324-4

Keeney, R.L. (1992). Value-focused thinking: a path to creative decisionmaking. Harvard University Press: Cambridge, Mass.

Keeney, R.L. and Raiffa, H. (1976). Decision with multiple objectives. Wiley, New York.

Korhonen, P., Wallenius, J., \& Zionts, S. (1984). Solving the discrete multiple criteria problem using convex cones. Management Science 30(11), 1336-1345. https://www.jstor.org/stable/2631568 Korhonen, P., Soleimani-damaneh, M. and Wallenius, J. (2016). Dual cone approach to convex-cone dominance in multiple criteria decision making. Eur. J. Oper. Res. 249, 1139-1143.

https://doi.org/10.1016/i.ejor.2015.09.043 
Korhonen, P., Soleimani-damaneh, M. and Wallenius, J. (2017). The use of quasi-concave value functions in MCDA: some theoretical results. Math. Meth. Oper. Res. 86, 367-375. https://doi.org/10.1007/s00186-017-0601-5

Langhans, S. D. and Reichert, P. (2011). Einbettung von Verfahren zur Fliessgewässerbewertung in ein übergeordnetes Gewässermanagementkonzept - Vorschläge am Beispiel des Modustufenkonzepts. Wasser Energie Luft 103(3), 204-214. https://www.dora.lib4ri.ch/eawag/islandora/object/eawag:6702

Langhans, S. D., Lienert, J., Schuwirth, N. and Reichert, P. (2013). How to make river assessments comparable: A demonstration for hydromorphology. Ecological Indicators 32, 264-275. https://doi.org/10.1016/i.ecolind.2013.03.027

Langhans, S.D., Reichert, P. and Schuwirth, N. (2014). The method matters: A guide for indicator aggregation in ecological assessments. Ecological Indicators 45, 494-507, 2014. https://doi.org/10.1016/j.ecolind.2014.05.014

Marttunen, M., Haag, F., Belton, V., Mustajoki, J. and Lienert, J. (2019). Methods to inform the development of concise objectives hierarchies in multi-criteria decision analysis. European Journal of Operational Research 277, 604-620. https://doi.org/10.1016/i.ejor.2019.02.039

Niederberger, K., Rey, P., Reichert, P., Schlosser, J., Helg, U., Haertel-Borer S. and Binderheim, E. (2016). Methoden zur Untersuchung und Beurteilung der Seen in der Schweiz; Modul Ökomorphologie Seeufer. Umwelt-Vollzug Nr. 1632, Swiss Federal Office of the Environment, Bern. https://www.dora.lib4ri.ch/eawag/islandora/object/eawag:16007

Prasad, S.Y., Karwan, M.H. and Zionts, S. (1997). Use of convex cones in interactive multiple objective decision making. Management Science 43(5), 723-734. https://doi.org/10.1287/mnsc.43.5.723

Ramesh, R., Karwan, M.A. and Zionts, S. (1988), Theory of convex cones in multicriteria decision making. Annals of Operations Research 16, 131-148. https://doi.org/10.1007/BF02283741

Reichert, P., Borsuk, M., Hostmann, M., Schweizer, S., Spörri, C., Tockner, K. and Truffer B. (2007). Concepts of decision support for river rehabilitation. Environmental Modelling \& Software 22, 188-201. https://doi.org/10.1016/j.envsoft.2005.07.017

Reichert, P., Schuwirth, N. und Langhans, S.D. (2011). MCWM - Ein Konzept für multikriterielle Entscheidungs-unterstützung im Wassermanagement. Wasser Energie Luft 103(2), 139-148. https://www.dora.lib4ri.ch/eawag/islandora/object/eawag:6688

Reichert, P., Schuwirth, N. and Langhans, S. (2013). Constructing, evaluating and visualizing value and utility functions for decision support. Environmental Modelling \& Software 46, 283-291. https://doi.org/10.1016/i.envsoft.2013.01.017 
Reichert, P., Langhans, S., Lienert, J. and Schuwirth, N. (2015). The Conceptual Foundation of Environmental Decision Support. Journal of Environmental Management 154, 316-332. https://doi.org/10.1016/i.jenvman.2015.01.053

Schlosser J. A., Haertel-Borer S., Liechti P. and Reichert P. (2013). Konzept für die Untersuchung und Beurteilung der Seen in der Schweiz. Anleitung zur Entwicklung und Anwendung von Beurteilungsmethoden. Bundesamt für Umwelt, Bern. Umwelt-Wissen Nr. 1326: $38 \mathrm{~S}$.

https://www.dora.lib4ri.ch/eawag/islandora/object/eawag:10780

Tversky, A., Kahneman, D. (1974). Judgement under uncertainty: heuristics and biases. Science 185, 1124-1131. https://doi.org/10.1126/science.185.4157.1124

Von Winterfeldt, D. and Edwards, W. (1986). Decision Analysis and Behavioural Research. Cambridge University Press. 